\title{
VARIACIÓN ESPACIO-TEMPORAL DE MICROALGAS ACUÁTICAS DEL EMBALSE DE BETANIA - HUILA Y SU RELACIÓN CON LA CALIDAD DEL AGUA
}

\author{
SPATIO-TEMPORAL VARIATION OF AQUATIC MICROALGAE OF THE EMBALSE DE \\ BETANIA-HUILA AND ITS RELATION WITH THE QUALITY OF WATER
}

\author{
Paula Martínez-Silva
}

\begin{abstract}
RESUMEN
El embalse de Betania es uno de los cuerpos de agua lénticos más importantes de Colombia, no solo por su importancia como generador de energía eléctrica sino por su uso como fuente de productividad económica a través de la piscicultura. Durante más de un año, se realizaron muestreos combinados de fitoplancton en diez estaciones del embalse de Betania. Las muestras se llevaron a un análisis cualitativo-cuantitativo, con el fin de poder establecer mediante el uso de índices ecológicos (Shannon-Weiver, Índice de Equidad de Pielou, Whilm y Dorris), el nivel de contaminación del agua y determinar si existe alguna fluctuación de ésta, entre las diferentes estaciones a lo largo del año. Los resultados permitieron concluir que el embalse de Betania presenta niveles de eutrofización, que se ven reflejados en la composición de organismos planctónicos registrados en los puntos de muestreo. Este proceso se intensifica cuando disminuyen las lluvias y coincide con una mayor mortandad de los peces. Es importante tomar medidas de manejo apropiadas, si se pretende prolongar la vida útil de este cuerpo de agua.
\end{abstract}

PALABRAS CLAVE: Fitoplancton, Índices Ecológicos, Análisis cualitativo y cuantitativo.

\begin{abstract}
The Betania reservoir is one of the most important lentic systems in Colombia, not only for its importance as generator of electric energy but also for being one of the main sources of economic productivity due to activities such as pisciculture. Over a period of one year, combined samples of phytoplankton were made in ten stations of the reservoir. The samples were studied under a quantitative-qualitative analysis in order to obtain three ecological indexes (Shannon-Weiver, Pielou and Whilm and Dorris) to establish the level of water pollution. An Analysis of Variance was applied to determine if there is any statistical fluctuation in the number of individuals per milliliter between stations or months. The results suggest that the Betania reservoir is going through an eutrophication process according to the organisms observed in the phytoplankton community. This process increases when rain decreases, concurrent with fish mortality episodes. It is necessary to take appropriate management measures in order to preserve the useful life of the reservoir.
\end{abstract}

KEY WORDS: phytoplankton, Ecological Indexes, Qualitative-Quantitative analysis.

\section{INTRODUCCIÓN}

Las algas de agua dulce constituyen un grupo sumamente diverso de organismos, que constituyen la base de la cadena trófica de muchos cuerpos de agua. Aunque pueden tener impactos positivos en los ecosistemas acuáticos, también pueden tener efectos adversos, así, por ejemplo cuando se presenta un alto número de algas se genera a continuación un proceso de descomposición que desencadena en la pérdida de oxígeno en el ecosistema, causando la muerte de los peces y otros problemas ecológicos. Por otra parte, algunas algas producen toxinas que son letales tanto para organismos acuáticos como para organismos terrestres. Es importante saber cuáles organismos generan impactos negativos cuando se monitorea un cuerpo de agua. En este orden de ideas las algas se pueden utilizar para mostrar y evaluar un amplio rango 
de impactos naturales y humanos en los ecosistemas acuáticos porque tienen una respuesta muy rápida a los cambios en el medio ambiente; algunos ejemplos de esto son la eutrofización por exceso de contaminación orgánica, polución industrial y cambios en el régimen hidrológico del cuerpo de agua en estudio (Bellinger y Sigee, 2010).

Los organismos indicadores de la calidad del agua determinan los efectos de los impactos en el ecosistema acuático a través de un tiempo prolongado. La información generada a partir de los Bioindicadores, no reemplaza los análisis fisicoquímicos, pero si reduce costos, por lo que estos estudios también son importantes en monitoreos de la calidad del agua (Chapman, 1996). De esta manera, el empleo de Bioindicadores es una técnica ecológica que se basa en la medición de la diversidad y presencia o ausencia de organismos específicos (De la Lanza et al., 2000).

En el embalse de Betania, desde su inundación, se han realizado varios estudios para evaluar su afectación en los ecosistemas acuáticos de los que hace parte, teniendo en cuenta especialmente las comunidades de peces y de plancton. Existen una amplia variedad de documentos al respecto, al igual que otra amplia gama evaluando sus características físico-químicas (Ramírez, 1993; Cala, 1995; Herrera y Guillot, 1999; CAM y ICA, 2007). La importancia de estudios que evalúen la variación espacio temporal de especies que puedan actuar con indicadoras de salud del ecosistema y específicamente para el área de estudio radica principalmente en que el Embalse de Betania constituye una de las principales fuentes del recurso pesquero en la región del Huila y por ende gran parte de la productividad económica del departamento dependen del buen mantenimiento y funcionamiento de este cuerpo de agua. Existen varios antecedentes de estudios similares en Colombia, incluyendo cuerpos de agua tanto lenticos como loticos e incluso algunas de las ciénagas más representativas del país (Roldán, 1992; Hernández-Atilano et al., 2005; Hernández-Atilano et al., 2008).

El presente documento muestra los resultados obtenidos a lo largo de un año de estudio (octubre 2013 - octubre 2014), sobre las especies de fitoplancton, presentes en el Embalse de Betania, obtenidas en diez puntos de muestreo. La idea de utilizar el fitoplancton, es que a parte de lo mencionado anteriormente, el fitoplancton contiene especies y géneros que son sumamente sensibles a la contaminación y géneros que son muy resistentes a la misma, permitiendo de esta manera ser empleados como Bioindicadores (Roldán, 1992; Ramírez,1993; Ramírez, 2000) de la calidad del agua presente en el embalse para generar un diagnóstico de la misma basado únicamente en este grupo Biológico que si bien no es el único si representa de manera confiable lo que a grosso modo puede estar sucediendo con el resto de comunidades Biológicas.

\section{MATERIALES Y MÉTODOS}

\section{Área de estudio}

El embalse Central Hidroeléctrica de Betania (CHB), está ubicado en la cuenca alta del río Magdalena, en el departamento del Huila $\left(02^{\circ} 42^{\prime}\right.$ Norte y $75^{\circ} 26^{\prime}$ Oeste) a 560 m de altitud (CHB, 1987; Cala, 1995; Figura 1). Está formado por el represamiento de los ríos Magdalena y Yaguará, cuenta con un área de inundación de 7400 ha, un volumen total aproximado de $1971106 \mathrm{~m}^{3}$, una profundidad máxima de $90 \mathrm{~m}$ y una vida útil de 50 años (Universidad Nacional de Colombia, 1986), su llenado inició en noviembre de 1986 y culminó en junio de 1987 (CHB, 1987).

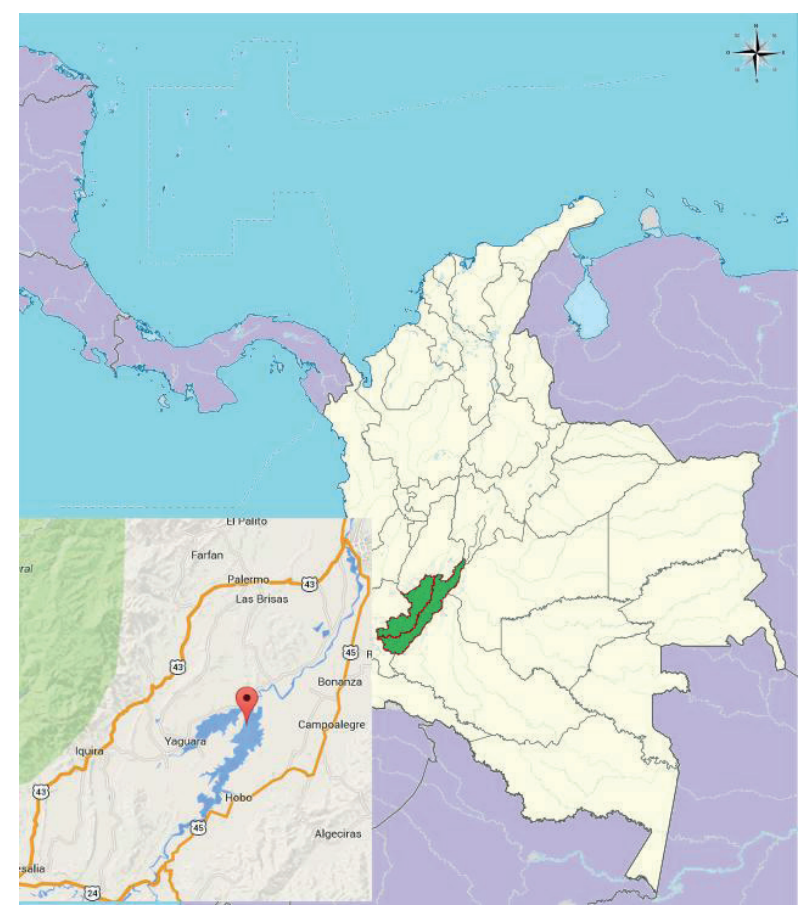

Figura 1. Localización del embalse de Betania en el Departamento del Huila, Colombia (Modificado de Google Maps ${ }^{\odot}$ ). 


\section{Muestreos}

Se ubicaron diez puntos de muestreo en el embalse, en su mayoría sobre estaciones piscícolas, los puntos o estaciones de muestreo fueron las siguientes: Estación 1: Embarcadero Hobo, Estación 2: Vega del Herrero, Estación 3: Cueva del Indio, Estación 4: ProcealBonanza, Estación 5: Ensenada Dique 6, Estación 6: María del Mar, Estación 7: Ensenada New York, Estación 8: Santa Helena, Estación 9: Presa Principal y Estación 10: Cola del Yaguará (Figura 2).

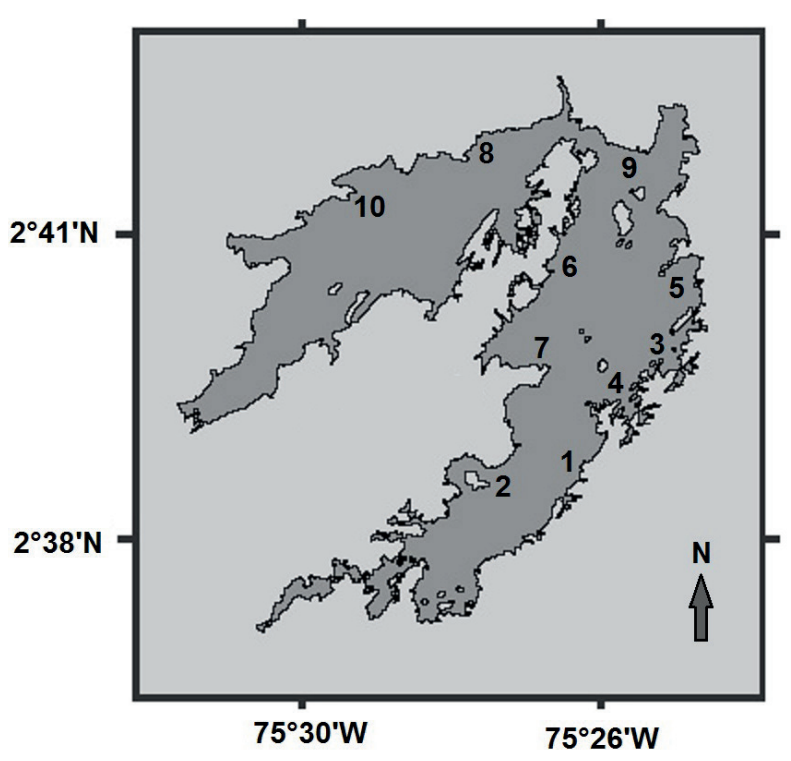

Figura 2. Estaciones de muestreo realizadas en el embalse de Betania.

Los muestreos se realizaron a cada dos meses uno del otro, entre agosto de 2013 y agosto de 2014. El fitoplancton se recolectó con un muestreo combinado, utilizando una botella Van-Dorn de 3 litros, con el cual se muestrearon los individuos de toda la columna fótica. Las muestras se fijaron en campo con solución Transeau y Lugol (Wetzel y Likens, 1979).

\section{Recuento e identificación}

Para el recuento e identificación del fitoplancton se siguió el método de Utermöhl (Vicente et al., 2005). Se utilizó un microscopio invertido de cuatro aumentos, una cámara de sedimentación de $100 \mathrm{~mm}^{3}$, unos formularios para anotar el recuento de las especies con una lista de taxones cuya presencia está comprobada en el embalse de Betania.
Cada muestra se dejó aclimatar por un periodo de 12 horas a temperatura ambiente, después fueron homogenizadas manualmente por un periodo de tres minutos, con la combinación de giros horizontales y verticales de la botella en la que estaban contenidas. Las submuestras se prepararon llenando la cubeta de sedimentación con la muestra, para lo cual se utilizó un volumen de muestra de $100 \mathrm{~mm}^{3}$ para el procedimiento. Las cubetas de sedimentación se cubrieron posteriormente con una pieza cuadrada de cristal, para evitar la formación de burbujas de aire. Cada cubeta de sedimentación se dejó en un lugar sin luz solar directa, a una temperatura constante de 24 ${ }^{\circ} \mathrm{C}$, durante 24 horas.

Las observaciones se hicieron utilizando un aumento de 40X, para el recuento se utilizó la cámara completa ya que habían muestras en la cuales la densidad de algas era muy baja.

La identificación del fitoplancton se hizo a nivel de especie en lo posible y a nivel de géneros cuando la identificación fue compleja y no se contó con la ayuda de expertos o con bibliografía especializada. Para el proceso se utilizaron las guías de identificación de Belcher y Swale (1978); Bicudo y Menezes (2006); Van Vuuren et al. (2006); Burgos y García (2007); Bellinger y Sigee (2010) y la base de datos de algas (http://www. algaebase.org/).

\section{Análisis de la información}

Para la validez estadística se tuvo como umbral que todas las muestras tuvieran como mínimo un número de individuos mayor o igual a 500, según Vicente et al. (2005). El cálculo de la concentración de fitoplancton se hizo con la siguiente ecuación: $\mathrm{N}=\mathrm{X}^{*}\left[\mathrm{~A} /\left(\mathrm{a}^{*} \mathrm{v}\right)\right]$; donde: $\mathrm{N}=$ número de células en la muestra $(\mathrm{cel} / \mathrm{ml})$, $\mathrm{X}=$ número medio de células por campo (o número total de células de la cámara), $\mathrm{A}$ = área de la cámara, $\mathrm{v}=$ volumen de muestra sedimentada en la cámara, a = área del campo óptico o de la cuadrícula (Modificado de Vicente et al., 2005).

Para el análisis ecológico de los resultados obtenidos se emplearon los índices de diversidad de Shannon-Weaver y de Equidad de Pielou, el cual se utilizó para evaluar el grado de similitud entre las estaciones y meses de muestreo (Magurran, 1988). Además se calculó el Índice de Wilhm y Dorris (1968), para evaluar el estado de contaminación en cada estación y por cada meses de muestreo. 
Los datos de densidad (individuos por mililitro), tanto por meses como por estaciones fueron sometidos a un análisis de varianza para lo cual primero se normalizaron con el fin de probar dos hipótesis nulas 1) Ho: No existe variación significativa entre las varianzas de número de individuos entre los diferentes meses en los que se tomaron las muestras; 2) Ho: No existe variación significativa entre las varianzas de número de individuos entre las diez estaciones en las que se tomaron las muestras. Se utilizó un nivel de confianza del $95 \%$ para los dos ANOVA. Este análisis se realizó en el programa estadístico QtiPlot v 0.9.9.2.

\section{RESULTADOS}

Se identificaron 60 especies de microalgas, agrupadas en 12 clases (Tabla 1). Las especies más abundantes fueron Synedra ulna, Ceratium hirudinella, Melosira granulata, Fragilaria sp. y Staurastrum leptocladum (Figura 3).

Tabla 1. Especies de algas acuáticas registrados para el embalse de Betania en el periodo 2013-2014.

\begin{tabular}{|c|c|c|c|c|}
\hline Clase & Orden & Familia & Género & Especie \\
\hline \multirow{6}{*}{ BACILLARIOPHYCEAE } & Cymbellales & Gomphonemataceae & Gomphonema & Gomphonema parvulum \\
\hline & Bacillariales & Bacillariaceae & Nitzschia & Nitzschia sp. \\
\hline & Cocconeidales & Cocconeidaceae & Cocconeis & Cocconeis placentula \\
\hline & Naviculales & Naviculaceae & Gyrosigma & Gyrosigma acuminatum \\
\hline & Naviculales & Pinnulariaceae & Pinnularia & Pinnularia biceps \\
\hline & Naviculales & Naviculaceae & Navicula & Navicula phyllepta \\
\hline \multirow{15}{*}{ CHLOROPHYCEAE } & Chlamydomonadales & Chlamydomonadaceae & Clamydomonas & Chlamydomonas sp. \\
\hline & Chlamydomonadales & Volvocaceae & Eudorina & Eudorina elegans \\
\hline & Chlamydomonadales & Volvocaceae & Pandorina & Pandorina morum \\
\hline & Sphaeropleales & Hydrodictyaceae & Pediastrum & Pediastrum clathratum \\
\hline & Sphaeropleales & Hydrodictyaceae & Pediastrum & Pediastrum duplex \\
\hline & Sphaeropleales & Hydrodictyaceae & Pediastrum & Pediasitrum simplex \\
\hline & Sphaeropleales & Hydrodictyaceae & Pediastrum & Pediastrum tetra \\
\hline & Sphaeropleales & Hydrodictyaceae & Tetraedron & Tetraedron trigonum \\
\hline & Sphaeropleales & Neochloridaceae & Golenkinia & Golenkinia sp. \\
\hline & Sphaeropleales & Scenedesmaceae & Coelastrum & Coelastrum reticulatum \\
\hline & Sphaeropleales & Scenedesmaceae & Scenedesmus & Scenedesmus acuminatus \\
\hline & Sphaeropleales & Scenedesmaceae & Scenedesmus & Scenedesmus ecornis \\
\hline & Sphaeropleales & Scenedesmaceae & Scenedesmus & Scenedesmus maximus \\
\hline & Sphaeropleales & Scenedesmaceae & Scenedesmus & Scenedesmus quadricauda \\
\hline & Chlamydomonadales & Volvocaceae & Volvox & Volvox aureus \\
\hline \multirow{5}{*}{ CONJUGATOPHYCEAE } & Desmidiales & Closteriaceae & Closterium & Closterium acutum \\
\hline & Desmidiales & Closteriaceae & Closterium & Closterium moniliferum \\
\hline & Desmidiales & Desmidiaceae & Arthrodesmus & Arthrodesmus octocornis \\
\hline & Desmidiales & Desmidiaceae & Cosmarium & Cosmarium bodrytis \\
\hline & Desmidiales & Desmidiaceae & Staurastrum & Staurastrum chaetoceras \\
\hline
\end{tabular}


Tabla 1. Continuación.

\begin{tabular}{|c|c|c|c|c|}
\hline Clase & Orden & Familia & Género & Especie \\
\hline \multirow{5}{*}{ CONJUGATOPHYCEAE } & Desmidiales & Desmidiaceae & Cosmocladium & Cosmocladium sp. \\
\hline & Desmidiales & Desmidiaceae & Staurastrum & Staurastrum teliferum \\
\hline & Desmidiales & Desmidiaceae & Staurastrum & Staurastrum leptocladum \\
\hline & Melosirales & Melosiraceae & Melosira & Melosíra granulata \\
\hline & Melosirales & Melosiraceae & Melosira & Melosíra ítalica \\
\hline \multirow{11}{*}{ CYANOPHYCEAE } & Chroococcales & Chroococcaceae & Chroococcus & Chroococcus turgidus \\
\hline & Chroococcales & Microcystaceae & Gloeocapsa & Gloeocapsa sp. \\
\hline & Chroococcales & Microcystaceae & Microcystis & Microcystis aeruginosa \\
\hline & Nostocales & Nostocaceae & Anabaena & Anabaena sphaerica \\
\hline & Synechococcales & Merismopediaceae & Merismopedia & Merismopedia sp. \\
\hline & Nostocales & Nostocaceae & Anabaena & Anabaena circinalis \\
\hline & Synechococcales & Merismopediaceae & Aphanocapsa & Aphanocapsa sp. \\
\hline & Gymnodiniales & Gymnodiniaceae & Gymnodinium & Gymnodinium excavatum \\
\hline & Peridiniales & Peridiniaceae & Peridinium & Peridinium cinctum \\
\hline & Oscillatoriales & Gloeotrichiaceae & Gloeotrichia & Gloeotrichia sp. \\
\hline & Synechococcales & Pseudoanabaenaceae & Pseudanabaena & Pseudanabaena sp. \\
\hline \multirow{2}{*}{ CHRYSOPHYCEAE } & Chromulinales & Dinobryaceae & Dinobryon & Dinobryon sp. \\
\hline & Hibberdiales & Stylococcaceae & Diceras & Diceras sp. \\
\hline \multirow{7}{*}{ GLENOPHYCEAE } & Euglenales & Euglenaceae & Euglena & Euglena acus \\
\hline & Euglenales & Euglenaceae & Euglena & Euglena oxyuris \\
\hline & Euglenales & Euglenaceae & Euglena & Euglena polymorpha \\
\hline & Euglenales & Euglenaceae & Trachelomonas & Trachelomonas acanthostoma \\
\hline & Euglenales & Euglenaceae & Trachelomonas & Trachelomonas acanthophora \\
\hline & Euglenales & Euglenaceae & Trachelomonas & Thachelomonas hispida \\
\hline & Euglenales & Euglenaceae & Trachelomonas & Trachelomonas volvocina \\
\hline \multirow{2}{*}{ FRAGILARIOPHYCEAE } & Fragilariales & Fragilariaceae & Fragilaria & Fragilaria sp. \\
\hline & Licmophorales & Ulnariaceae & Synedra & Synedra ulna \\
\hline \multirow{2}{*}{ DINOPHYCEAE } & Gonyaulacales & Ceratiaceae & Amphiceratium & Amphiceratium sp. \\
\hline & Gonyaulacales & Ceratiaceae & Ceratium & Ceratium hirudinella \\
\hline MEDIOPHYCEAE & Thalassiosirales & Stephanodiscaceae & Cyclotella & Cyclotella meneghiniana \\
\hline \multirow{3}{*}{ TREBOUXIOPHYCEAE } & Chlorellales & Chlorellaceae & Dictyosphaerium & $\begin{array}{l}\text { Dictyosphaerium } \\
\text { ehrenbergianum }\end{array}$ \\
\hline & Chlorellales & Chlorellaceae & Dictyosphaerium & Dictyosphaerium pulchellum \\
\hline & Trebouxiales & Botryococcaceae & Botryococcus & Botryococcus braunii \\
\hline SYNUROPHYCEAE & Synurales & Mallomonadaceae & Mallomonas & Mallomonas sp. \\
\hline
\end{tabular}



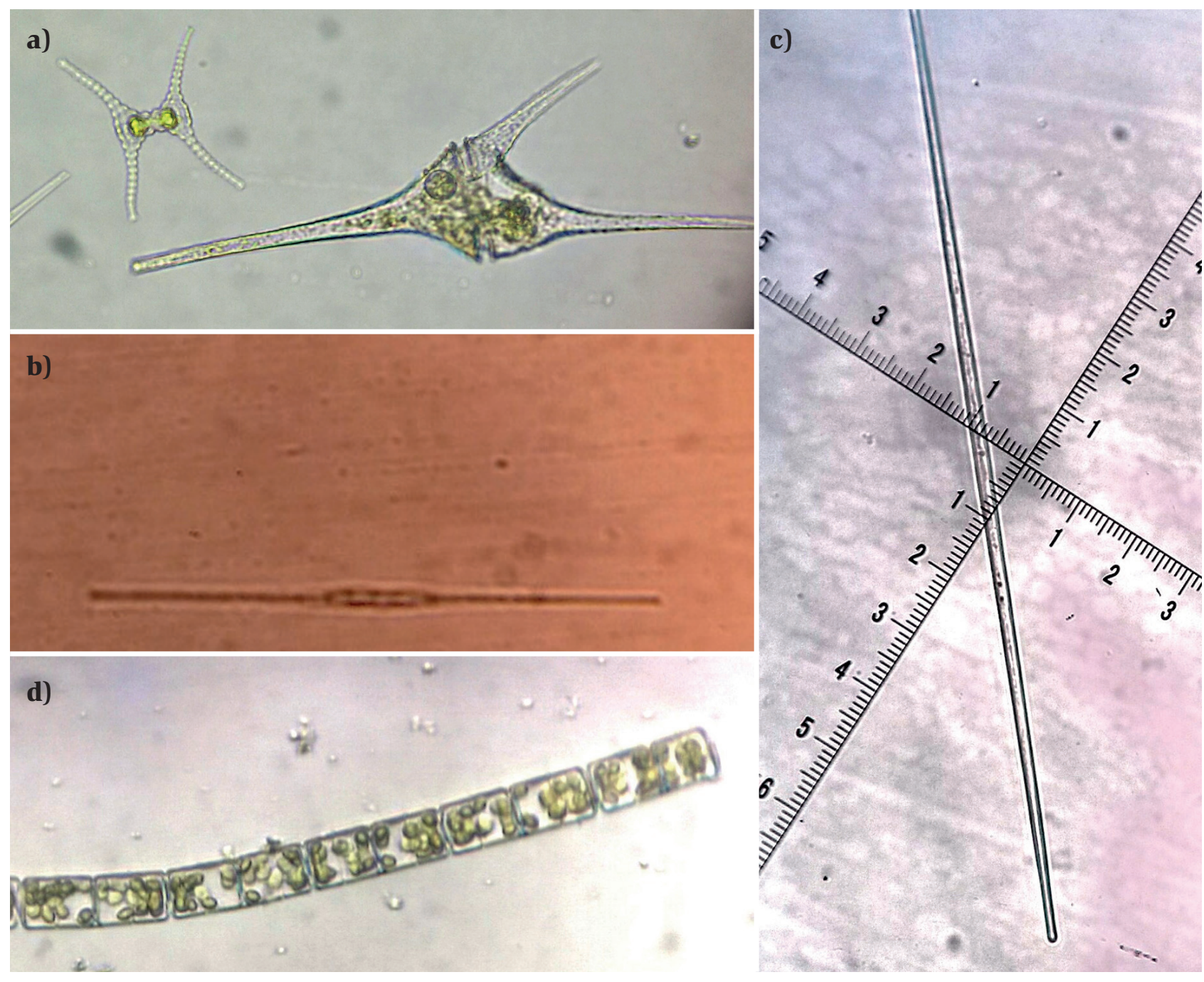

Figura 3. Especies de algas más abundantes en el embalse de Betania entre agosto de 2013 y agosto de 2014. a) Ceratium hirudinella y Staurastrum leptocladum. b) Fragilaria sp. c) Synedra ulna. d) Melosira granulata.

Los valores de los índices ecológicos por mes y por estación de muestreo, se muestran en las Tablas 2 y 3 respectivamente. En los meses de diciembre de 2013 y febrero de 2014, para las diez estaciones, se registraron los mayores valores de abundancias (Tabla 4). Específicamente las estaciones 3, 4 y 5 , presentaron las mayores concentraciones de individuos durante los muestreos.
Según el análisis de varianza, no se encontraron diferencias significativas entre la concentración de individuos en los meses de muestreo ( $\mathrm{F}=0,33632$, $\mathrm{P}=0,91751)$. De igual manera para la hipótesis nula 2, no se registraron diferencias significativas entre la concentración de individuos en las estaciones de muestreo ( $\mathrm{F}=0,12986, \mathrm{P}=0$,99891). 
Tabla 2. Resultados de los índices ecológicos calculados para cada mes de muestreo.

\begin{tabular}{|c|c|c|c|c|c|c|c|}
\hline & AGOSTO 2013 & OCTUBRE 2013 & DICIEMBRE 2013 & FEBRERO 2014 & MAYO 2014 & JULIO 2014 & AGOSTO 2014 \\
\hline $\begin{array}{l}\text { INDICE DE } \\
\text { SHANNON- } \\
\text { WEAVER } \\
\end{array}$ & 1,989 & 0,870 & 1,139 & 1,358 & 1,534 & 3,046 & 2,709 \\
\hline $\begin{array}{l}\text { ÍNDICE DE } \\
\text { EQUIDAD DE } \\
\text { PIELOU }\end{array}$ & 0,569 & 0,321 & 0,363 & 0,433 & 0,666 & 0,971 & 0,831 \\
\hline $\begin{array}{l}\text { INDICE DE } \\
\text { WILHM AND } \\
\text { DORRIS }\end{array}$ & 1,379 & 0,603 & 0,789 & 0,941 & 1,063 & 2,111 & 1,878 \\
\hline
\end{tabular}

Tabla 3. Resultados de los índices ecológicos calculados para cada estación.

\begin{tabular}{|c|c|c|c|c|c|c|c|c|c|c|}
\hline & $\begin{array}{r}\text { Embarcadero } \\
\text { Hobo }\end{array}$ & $\begin{array}{r}\text { Vega del } \\
\text { Herrero }\end{array}$ & $\begin{array}{r}\text { Cueva del } \\
\text { indio }\end{array}$ & $\begin{array}{c}\text { Proceal- } \\
\text { Bonanza }\end{array}$ & $\begin{array}{r}\text { Ensenada } \\
\text { Dique } 6\end{array}$ & $\begin{array}{r}\text { Maria } \\
\text { del mar }\end{array}$ & $\begin{array}{l}\text { Ensenada } \\
\text { New York }\end{array}$ & $\begin{array}{r}\text { Santa } \\
\text { Helena }\end{array}$ & $\begin{array}{r}\text { Presa } \\
\text { Principal }\end{array}$ & $\begin{array}{l}\text { Cola del } \\
\text { Yaguará }\end{array}$ \\
\hline $\begin{array}{l}\text { ÍNDICE DE } \\
\text { SHANNON- } \\
\text { WEAVER }\end{array}$ & 1,516 & 1,128 & 1,464 & 1,525 & 1,639 & 1,786 & 2,289 & 2,245 & 1,470 & 1,763 \\
\hline $\begin{array}{l}\text { ÍNDICE DE } \\
\text { EQUIDAD DE } \\
\text { PIELOU }\end{array}$ & 0,471 & 0,332 & 0,426 & 0,316 & 0,322 & 0,310 & 0,319 & 0,325 & 0,341 & 0,329 \\
\hline $\begin{array}{l}\text { ÍNDICE DE } \\
\text { WILHM AND } \\
\text { DORRIS }\end{array}$ & 1,051 & 0,782 & 1,015 & 1,057 & 1,136 & 1,238 & 1,586 & 1,556 & 1,019 & 1,222 \\
\hline
\end{tabular}

Tabla 4. Resultados de conteo total de número de individuos por mililitro por estación.

Estación 1 Estación 2 Estación 3 Estación 4 Estación 5 Estación 6 Estación 7 Estación 8 Estación 9 Estación 10

Número

$\begin{array}{llllllllll}\text { Total de } & 36234 & 56130 & 96086 & 102089 & 102126 & 49258 & 29278 & 45552 & 59497\end{array}$

Ind.

\section{DISCUSIÓN}

Las estaciones con mayor concentración de individuos por mililitro fueron la Cueva del Indio, Proceal-Bonanza y Ensenada Dique 6, las cuales corresponden a estaciones donde la velocidad del agua es baja, el espejo de agua es pequeño y la producción de tilapias el alta, factores que facilitan que las comunidades de algas se puedan establecer y crecer.

Las especies más abundantes encontradas en el embalse, corresponden a algas que en general se desarrollan 
mejor en condiciones eutróficas, lo cual va acorde con las condiciones actuales del embalse de Betania, donde se ha registrado que la calidad del agua disminuye poco a poco, no solo por la contaminación de las aguas de los ríos que lo surten, sino por las actividades de ganadería y agricultura en los alrededores y por las prácticas de piscicultura que se desarrollan en el embalse a través de los años. Por otro lado la mayoría de las especies encontradas, corresponden a organismos indicadores de Betamesosaprobiedad y de Oligosaprobiedad, con valores de saprobiedad entre 1 y 2,5, lo que muestra que el agua está ligeramente sucia pero sirve para agua de riego y pesca (De la Lanza et al., 2000).

Los valores de los índices ecológicos para los meses de octubre de 2013 y febrero de 2014, mostraron valores bajos, lo que se traduce en indicadores de alta contaminación, mientras que los meses de julio y agosto presentaron los menores niveles de contaminación. Este hecho lo corrobora los valores presentados en la tabla 1 para los meses diciembre de 2013 y febrero de 2014, en donde se mostró una mayor concentración de individuos por mililitro representados en mayor medida por especies de las cuales se ha documentado que indican altos niveles de contaminación. Por otro lado, al observar las tendencias anuales del embalse muestran una periódica mortandad de peces entre los meses de febrero y abril, lo cual refleja esta situación. Varias de las especies de microalgas encontradas son tóxicas para los peces, como es el caso de especies de los géneros Ceratium, Microcystis y Pandorina.

Los valores de los índices para las estaciones, muestran niveles de moderados a muy altas de contaminación, los cuales se acentuaron en los meses que disminuyó la precipitación. Los valores de índice de Shannon-Weaver que se muestran en la tabla 2, son consistentes con los problemas de eutrofización del agua. Las estaciones de Vega del Herrero, Cueva del Indio y Presa Principal, son las más afectadas, resultado que puede estar ligado a que el agua tiene menos velocidad, lo que permite que se acumulen más residuos, lo que ocaciona, como se dijo anteriormente, que las comunidades de algas tengan más tiempo para establecerse.

Para las demás estaciones donde la velocidad de 18 circulación del agua es más rápida y por ende el recambio de nutrientes es mayor (ej. Estaciones Ensenada New York, Santa Helena), los valores de los índices fueron menores y por las condiciones mencionadas el tiempo para que se establezcan las comunidades de algas es menor. El patrón observado para los valores del índice de Shannon-Weaver, se mantienen al analizar los resultados del índice de Whilm y Dorris, donde en estaciones como Ensenada New York y Santa Helena se presume valores bajos o moderados de contaminación.

Con el análisis de varianza se pudo observar que no existe una diferencia significativa entre las varianzas del número de individuos encontrados por mililitro, tanto entre estaciones como entre meses, lo cual refuerza nuevamente el hecho de que existe una tendencia generalizada del embalse hacia la eutrofización.

Los resultados refuerzan la tendencia observada y documentada en el embalse, donde el establecimiento y acelerado crecimiento de comunidades algales y el proceso de eutrofización se acentúan cada vez más, lo que conlleva a un aumentando de los niveles de saprobiedad. De continuar estas tendencias las empresas piscícolas se verán afectadas drásticamente en su actividad no van a poder continuar desarrollando sus actividades y se verá diezmado uno de los principales ingresos o motores de la economía Huilense.

\section{CONCLUSIONES}

Existe una tendencia clara del embalse a un proceso de eutrofización continuo lo cual se refleja en las comunidades planctónicas que lo habitan.

Los niveles de eutrofización aumentan en las épocas de lluvias bajas, y esto a su vez, está relacionado con un aumento en el nivel de mortalidad de los peces del embalse.

Es importante tomar medidas al respecto si se pretende que la vida útil del embalse de Betania se prolongue, porque de no tomarse medidas al respecto el departamento del Huila puede perder no solo su recurso energético sino también una de las principales fuentes de productividad económica.

Se recomienda hacer estudios de fitoplancton en intervalos de tiempo más cortos, puesto que los ciclos de vida de las algas son muy cortos y espacios de muestreo de dos meses pueden no estar reflejando con exactitud lo que ocurre con las comunidades de algas. 


\section{BIBLIOGRAFÍA}

Belcher, H. y Swale, E. 1978. A beginner's guide to Freshwater algae. Institute of Terrestrial Ecology. Natural Environment Researrch Council. London.

Bellinger, C.G. y Sigee, D.C. 2010. Freshwater algae: Identification and use as bioindicators. Wiley-Blackwell. London, UK.

Bicudo, C.E. y Menezes, M. 2006. Géneros de algas de aguas continentales del Brasil: Clave para la identificación y descripciones. Segunda Edición. RiMa Editorial. Brasil.

Burgos, M.D. y García, E. 2007. Atlas de Microorganismos presentes en los humedales Andaluces. Consejo del Medio Ambiente, Junta de Andalucía, España.

Cala, P. 1995. Trophic levels of the most abundant fishes of the Betania reservoir, upper Río Magdalena, Colombia. Acta Biológica Venezolana 16: 47-5 3.

CAM y ICA. 2007. Informe sobre la crisis que se presentó en la piscicultura que se desarrolla en Betania y estado del arte de esta apuesta productiva promisoria. Huila. Corporación Autónoma del Alto Magdalena https://www.ccneiva.org/ imagenes/File/INFOPISCICULTURA.pdf

C.H.B. (Central Hidroeléctrica de Betania). 1987. Informe de llenado del embalse. C.H.B. Bogotá.

Chapman, D. 1996. Water quality assessments: A guide to the use of Biota, sediments and water in environmental monitoring. Chapman Hill, Londres.

De La Lanza, E.G., Hernández, P.S. y Carbajal, J.P.L. 2000. Organismos indicadores de la calidad del agua y de la contaminación, Bioindicadores. Plaza y Valdés. México.

Hernández-Atilano, E., Aguirre, N.J., Palacio, J.A. y Ramírez-Restrepo, J.J. 2008.Variación espacio temporal de la asociación fitoplanctónica en diferentes momentos del pulso hidrológico en la ciénaga de Ayapel (Córdoba). Actualidades Biológicas 30(88): 67 - 81.
Hernández-Atilano, E., Aguirre, N.J. y Palacio, J.A. 2005. Variación Espacio-temporal de la estructura de la comunidad de algas perfíticas en la microcuenca de la Quebrada La Vega, Municipio de San Roque (Antioquia) Colombia. Actualidades Biológicas 27(82): 67 - 77.

Herrera, M. y Guillot, G. 1999. Composición taxonómica del zooplancton del embalse de Betania, departamento del Huila, Colombia. Acta Biológica Colombiana 4(1): 5-19.

Magurran, A. 1988. Ecological Diversity and its measurement. Chapman and Hall, London.

Ramírez, J.J. 2000. Fitoplancton de agua dulce: aspectos ecológicos, taxonómicos y sanitarios. Editorial Universidad de Antioquia. Medellín, Colombia.

Ramírez, M.E. 1993. Contribución al estudio del fitoplancton en el embalse de Betania, Huila. Trabajo de grado, Departamento de Biología, Facultad de Ciencias, Universidad Nacional de Colombia. Santa fe de Bogotá.

Roldán, P.G. 1992. Fundamentos de Limnología Neotropical. Primera edición. Editorial Universidad de Antioquia. Colección Ciencia y Tecnología U de A. Medellín, Colombia.

Universidad Nacional De Colombia. 1986. Diagnóstico Ambiental del Impacto del Proyecto Hidroeléctrico de Betania. Universidad Nacional de Colombia, Facultad de Ciencias. Bogotá.

Van Vuuren, S.J., Taylor, J., Van Ginkel, C. y Gerber, A. 2006. Easy identification of the most common freshwater algae. A guide for the identification of microscopic algae in South African freshwaters. School of Environmental Sciences and development. Botany. North-West University of South Africa.

Vicente, E., De Hoyos, C., Sánchez, P. y Cambra, J. 2005 Metodología para el establecimiento del estado Ecológico según la directiva Marco del Agua: Protocolo de Muestreos y Análisis para Fitoplancton. Confederación Hidrográfica del Ebro. España.

Wetzel, R.G. y Likens, E.G. 1979. Limnologycal analysis. W.B. Saunders Co., London.

Fecha de recepción: 13/04/2015 Fecha de aceptación: 25/08/2015

Para citar este artículo: Martínez-Silva, P. 2015. Variación espacio-temporal de microalgas acuáticas del embalse de Betania - Huila y su relación con la calidad del agua. Revista Intropica Vol. 10: 11 - 19 